\title{
Winter: A Novel Low Power Modular Platform for Wearable and IoT Applications
}

\author{
Patrick Locatelli ${ }^{1(凶)}$, Asad Hussain ${ }^{1}$, Andrea Pedrana $^{1}$, Matteo Pezzoli ${ }^{2}$, \\ Gianluca Traversi ${ }^{1}$, and Valerio $\operatorname{Re}^{1}$
}

${ }^{1}$ Department of Engineering and Applied Sciences, University of Bergamo, Viale Marconi 5, 24044 Dalmine, BG, Italy

patrick.locatelli@unibg.it

2 Department of Electrical, Computer, and Biomedical Engineering, University of Pavia,

Via Ferrata 5, 27100 Pavia, PV, Italy

\begin{abstract}
This paper presents a new multi-purpose, ultra-low power device designed to cope with the typical limitations of commercial IoT platforms. The device can be used both as (1) a stand-alone system, with embedded sensing, data processing, storage and communication capabilities, and as (2) a motherboard for miniaturized expansion boards, which can be plugged on its top to enhance the basic features. The paper also provides the performances of the system, as well as a use case in an IoT context.
\end{abstract}

\section{Introduction}

Thanks to the synergy of wireless technologies, Micro Electro-Mechanical Systems (MEMS) and the Internet, smart and wearable devices are becoming the driving force in the Internet-of-Things (IoT) era. The capability to monitor different parameters by means of several sensing units enables such systems to a wide range of applications: from the recording of human movements for fitness and rehabilitation purposes, to the monitoring of environmental parameters for quality of life assessment [1].

In this context, the massive spread of machine learning and big data applications amazingly increased not only the amount, but also the variety of data needed. This is especially true for medical research projects, in which the selection of the optimal devices has taken on a key role [2]. It is the authors' opinion that among the requirements such devices should address, the availability of raw data which can be processed by in-house algorithms, and the opportunity to collect different data types for long periods are the most valuable for research purposes. Nevertheless, most of the commercial devices do not grant access to the raw information, providing only the output of proprietary algorithms; on the other hand, many research-graded products are designed for specific applications, with either low memory or few sensors.

Driven by the limitations of the wearable devices used in previous studies, the authors developed a new wearable platform, specifically designed to be both (1) an efficient monitoring system suitable for edge computing and real-time data analysis, and (2) a long-term data logger, providing up to a few days of continuous data log thanks to low power consumption and a high-capacity memory. 


\section{System Architecture}

Winter, an acronym of Wearable Inertial TrackER, is the result of a design aimed to provide a system-on-board with sensing and processing capabilities, low power consumption, (relatively) high storage capacity thanks to an on-board SD connector and wireless transmission capabilities provided by a BLE module, all in a form factor of $32 \times 20 \mathrm{~mm}^{2}$ (see Fig. 1). The overall block architecture of the system, as well as the communication interfaces used, are depicted in Fig. 2.

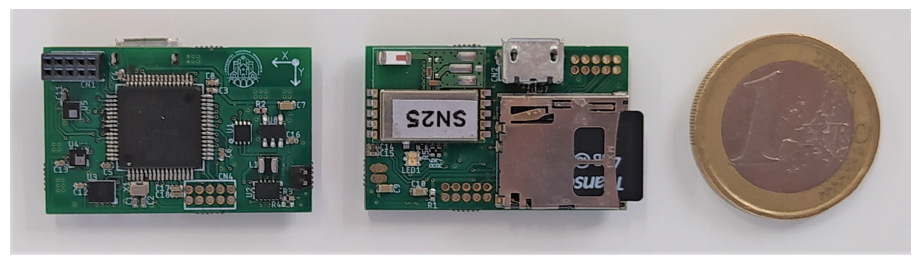

Fig. 1. The winter device.

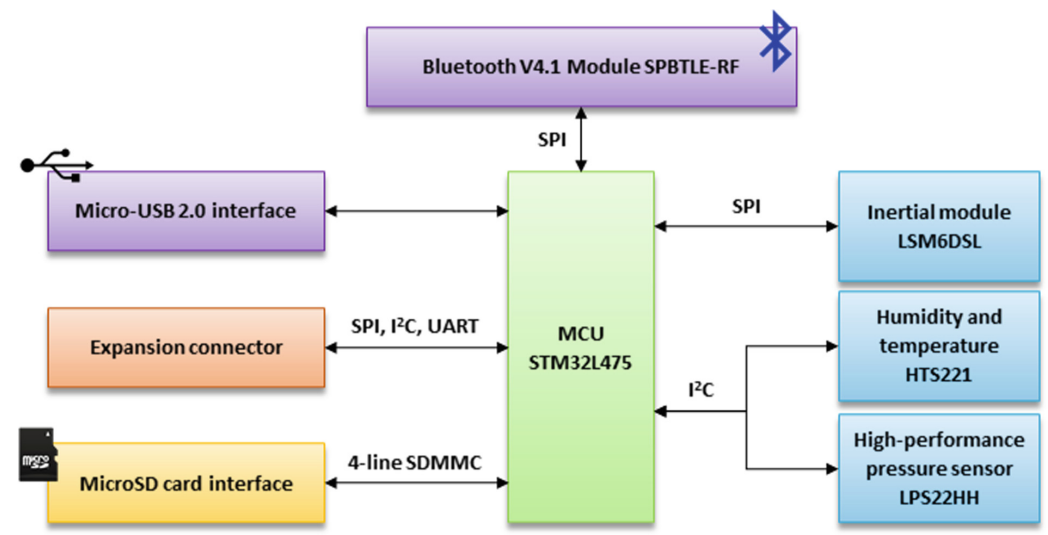

Fig. 2. Block diagram of the winter platform. processing, sensing, storage, connectivity and I/O blocks are grouped by color.

Processing. The core processing unit of Winter is the ultra-low power STM32L475RG microcontroller unit (MCU), manufactured by STMicroelectronics. Based on the ARM Cortex-M4 32-bit architecture, it embeds high-speed memories (1 MB of Flash memory, $128 \mathrm{kB}$ of SRAM), a low-power RTC (Real-Time Clock) and an extensive range of enhanced I/Os and peripherals. In addition to this, the ABS06-1-T SMD crystal (Abracon) was connected to the microcontroller: by generating a clock signal of $32.768 \mathrm{kHz}$ with a frequency tolerance of $\pm 10 \mathrm{ppm}$ (parts per million), a more accurate and precise timebase can be obtained.

Sensing. The sensing capabilities of Winter range from environmental monitoring to inertial measurements. These are achieved by the presence of three on-board modules. 
The first module is the LSM6DSL (STMicroelectronics), a system-in-package featuring a 3D digital accelerometer and a 3D digital gyroscope with full-scale ranges of $\pm 2 / \pm 4 / \pm 8 / \pm 16 \mathrm{~g}$ and $\pm 125 / \pm 245 / \pm 500 / \pm 1000 / \pm 2000 \mathrm{dps}$, respectively. The module has a current draw of $0.65 \mathrm{~mA}$ when both sensors set to operate in high-performance mode, and it enables always-on low-power features. An SPI connection to the MCU was preferred over the $\mathrm{I}^{2} \mathrm{C}$ bus to work at higher baud rates.

The second and third sensing modules are the HTS221 and the LPS22HH, from STMicroelectronics. The former is an ultra-compact sensor for relative humidity $(\mathrm{rH})$ and temperature, with an accuracy of $\pm 3.5 \%$ in the $20-80 \% \mathrm{rH}$ interval and $\pm 0.5{ }^{\circ} \mathrm{C}$ in the temperature range from 15 to $40{ }^{\circ} \mathrm{C}$. The latter is an ultra-compact piezoresistive absolute pressure sensor functioning as a digital output barometer, with a 260 to $1260 \mathrm{hPa}$ absolute pressure range (accuracy: $0.5 \mathrm{hPa}$ ). Both modules are connected to the MCU via an $\mathrm{I}^{2} \mathrm{C}$ interface and are suitable for ultra-low power applications, having a current draw below $3 \mu \mathrm{A}$ at $1 \mathrm{~Hz}$ ODR (Output Data Rate).

Storage. The MicroSD card socket mounted on the Winter platform makes it possible to use an external memory card to store raw data and processed results. The data transfer is performed by using the 4-line SDMMC communication interface provided by the MCU, at a rate up to $48 \mathrm{MHz}$ (8-bit mode). The stored data can be accessed either by removing the SD card or through the USB connection.

Connectivity. The communication with external devices allows Winter (1) to transfer the output of the embedded data analysis algorithms, and (2) to be remotely configured to operate in one of the available working modes. To enable such communication, two different solutions were adopted. The first solution is represented by the SPBTLE-RF, an easy-to-use Bluetooth Low Energy master/slave network processor module provided by STMicroelectronics, compliant with Bluetooth v4.1. On the other hand, Winter provides a Micro-USB 2.0 high-speed port that allows data transfer with an external device in both directions.

Power Management. The system is powered by a $3.7 \mathrm{~V}, 210 \mathrm{mAh}$ lithium polymer battery, scaled down to the $3 \mathrm{~V}$ operating voltage by means of a high efficiency stepdown converter (TPS62740 manufactured by Texas Instruments). The battery can be recharged through the dedicated micro-USB connector and the process is supervised by the MCP73831 charging controller by Microchip. To monitor the battery charging level, the MAX17048X + was integrated and connected to the MCU via $\mathrm{I}^{2} \mathrm{C}$ interface.

I/O. Two 10-pin connectors are mounted on the bottom side of the Winter platform. The first one is a simple debug connector meant to be removed at release stage. The second one enables the Winter platform to extend its sensing and communication capabilities by providing a socket for potential expansion boards. In fact, the system's power supply, as well as all of the most common interfaces available (SPI, $\mathrm{I}^{2} \mathrm{C}$, UART) can be exploited through this connector by any expansion board, which only needs to be plugged on the top of Winter. In such a context, while the Winter platform would act as a motherboard, i.e. supplying the required power and providing basic storage and connectivity capabilities, an expansion board could be designed with the strictly necessary components, thus minimizing its form factor. 


\section{Firmware and Performance}

\subsection{Finite-State Machine}

Figure 3 depicts the finite-state machine (FSM) embedded on the Winter's MCU, whereas the state details are described in Table 1.

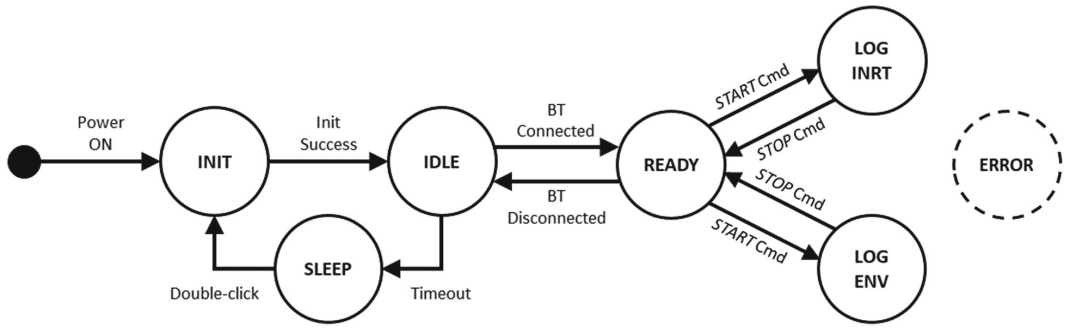

Fig. 3. Finite-state machine of the winter platform.

Table 1. List of Winter's FSM states (Inrt: inertial; Axl: accelerometer; Env: environmental).

\begin{tabular}{l|l|l|l|l|l}
\hline Name & MCU mode & Inrt sensors & Env sensors & Data storage & Bluetooth status \\
\hline INIT & Run & - & - & - & - \\
\hline IDLE & LP Run & OFF & ON & Inactive & Advertising \\
\hline SLEEP & Stop 2 & Axl ON & OFF & OFF & OFF \\
\hline READY & LP Run & OFF & ON & Inactive & Connected \\
\hline LOG_INRT & LP Run/Run & ON & ON & Active & Connected/Adv \\
\hline LOG_ENV & Run/Stop 2 & OFF & ON & Active & Connected/Adv \\
\hline
\end{tabular}

After the success of the startup routine (INIT state), the platform enters the IDLE state characterized by the inertial sensor turned off, the environmental sensing components powered on and the Bluetooth (BT) module being ready to accept a connection. If a connection is not established within a defined time window $(1 \mathrm{~min})$, the system enters the SLEEP state: the BT module is switched off and the microcontroller is set to operate in Stop 2 mode. A double-click event detected by the inertial module awakens the system in the INIT state.

From the IDLE state, an active BT connection makes the FSM move to the READY state, in which the platform is ready to communicate with the master device. By means of the BT command set, Winter can then enter two different log states. The first one is called LOG_INRT, and involves the log of inertial data from the selected sensors (only accelerometer, only gyroscope or both) on the SD card at a selected frequency; the MCU continuously alternates the Low-Power Run mode (collection phase) and the Run mode (SD writing/analysis phase) to reduce the mean power consumption. The second one is called LOG_ENV: while the inertial sensors are turned off, the MCU is kept in Stop 
2 mode and is awaken (Run mode) every 1 min only for the time required to read the environmental parameters and store them on the SD card. Both these states can operate either with an active BT connection, or with the BT module in Advertising mode.

Data collected by Winter can be accessed in two ways only. The first one requires the user to remove the SD card: all the logged data (either inertial or environmental, depending on the mode previously used) can be downloaded to any device embedding an SD support (e.g. computer, smartphone), and the SD card can then be cleared and used again for new acquisition sessions. Alternatively, data are periodically provided to the outer world through the BLE characteristics system: this holds true only for environmental data, and it is performed in all those states in which such sensors are active. No real-time data streaming is currently provided by the system, although it could be implemented in future releases of the firmware.

During each of the different states of the FSM, the scheduling of tasks to perform is based on the internal interrupts handling mechanism natively embedded in the MCU management. For each task, the timebase represented by the system clock is used to raise some internal interrupts after a specific time period elapsed; then, the related call-back function is invoked depending on the interrupt priority (i.e. the priority of the task); finally, the code related to the interrupt handling is executed. Specifically, the main tasks performed by the system are the following (the order defines the priority from highest to lowest): (1) reading the data collected by the on-board sensors from the respective registers; (2) logging the data onto the SD card (if present); (3) handling the Bluetooth connection, by interpreting the incoming commands and by updating the characteristics to be read from the user.

\subsection{Power Consumption}

By means of the developed testing firmware, a preliminary evaluation of the overall power consumption was carried out. The results of the measurements are reported in Table 2 . The 210 mAh battery allows up to 3 days of continuous inertial data log (with both accelerometer and gyroscope's ODRs set to $416 \mathrm{~Hz}$ ), or up to 2 months of environmental monitoring (with one-shot measurements per minute).

Table 2. Average power consumption of the states (power supply: $3.7 \mathrm{~V}, 210 \mathrm{mAh}$ battery).

\begin{tabular}{l|l|l|l}
\hline State & Current drawn & $\begin{array}{l}\text { Power } \\
\text { consumption }\end{array}$ & Autonomy \\
\hline IDLE & $0.966 \mathrm{~mA}$ & $3.574 \mathrm{~mW}$ & 9 days \\
\hline SLEEP & $0.045 \mathrm{~mA}$ & $0.167 \mathrm{~mW}$ & 6 months \\
\hline READY & $1.046 \mathrm{~mA}$ & $3.870 \mathrm{~mW}$ & 8 days \\
\hline LOG_INRT & $2.881 \mathrm{~mA}$ & $10.660 \mathrm{~mW}$ & 3 days \\
\hline LOG_ENV & $0.171 \mathrm{~mA}$ & $0.633 \mathrm{~mW}$ & $\sim 2$ months \\
\hline
\end{tabular}




\subsection{Data Comparison}

Accelerations collected by Winter were compared to those collected by MuSe, a researchgrade inertial platform used by authors in other works [3, 4], both in static and dynamic conditions (Fig. 4). Acceleration signals collected by the two platforms are almost identical on each axis, thus confirming the possibility to use Winter in place of the MuSe one when the collection of inertial data is needed. Some negligible differences can still be observed in static conditions: this can be ascribed to the different $0 \mathrm{~g}$-offset values of the devices' inertial modules ( $\pm 90 \mathrm{mg}$ for MuSe's LSM9DS1, $\pm 40 \mathrm{mg}$ for Winter's LSM6DSL). However, such differences will be reduced once the self-zeroing procedure will be implemented.
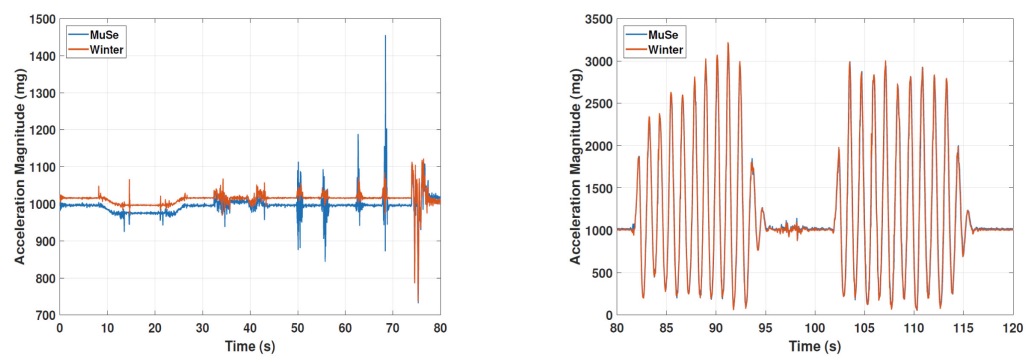

Fig. 4. Comparison of acceleration magnitude collected by MuSe and Winter devices in quasistatic (left) and dynamic (right) conditions.

\section{IoT Application: Indoor Environmental Monitoring}

To simultaneously test the system and to provide a use case of the Winter platform, an IoT-oriented application was developed. Specifically, the presented device was used to continuously collect information about the environmental conditions of a room, i.e. temperature, humidity level and atmospheric pressure. The system is shown in the lefthand side of Fig. 4 and was composed of: (1) a Winter device, used to collect the environmental data; (2) a Raspberry Pi 3, connected to the Internet via an Ethernet cable and acting as a gateway; (3) an open-source IoT application named ThingSpeak, which provides both a cloud server and a customizable graphical front-end.

The availability of a BLE module on the Raspberry platform allowed a Python script to be developed ad-hoc for a periodical readout of the environmental data: these were made available by Winter as characteristics of the standard Environmental Sensing Service (ESS) of the BLE protocol. Data were then streamed to ThingSpeak cloud to aggregate and visualize the live values. Towards this goal, a dedicated channel was created on the web platform at first; then, the provided API keys as well as the function calls were embedded in the aforementioned Python script, to allow the batch job to send data packets via the HTTP protocol: Fig. 5 (right) depicts ThingSpeak's temperature chart for multiple monitoring sessions. Starting from this simple example, a network composed of several Winter nodes connected to the same gateway could be easily set up to monitor different rooms within the same building. 

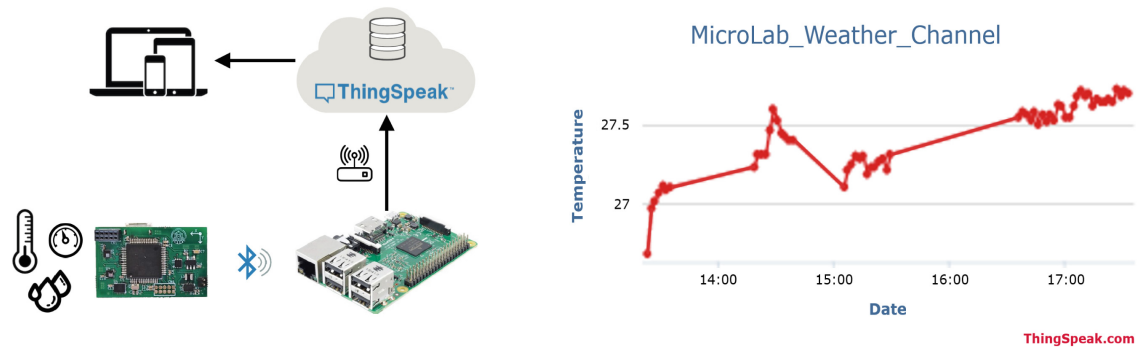

Fig. 5. On the left, the environmental monitoring system based on Winter device. On the right, a web chart representing the output of the embedded temperature sensor.

\section{Conclusions}

This work presented a new multi-purpose, smart, wearable device specifically designed to overcome the limitations of the typical devices used in IoT contexts. Performance tests confirmed the capability of the platform to work in ultra-low power regimes. Moreover, the similarities between inertial data from Winter and MuSe support the replacement of the latter in all the authors' past (or future?) studies. An application example was also described for Winter: the device was used as an IoT node for environmental monitoring. However, the variety of integrated sensors and the enhancement capabilities supported by the expansion connector allows Winter to be used for many purposes: activity tracking, home rehabilitation assistance, physiological monitoring and quality of life assessment are just some of the potential applications.

Acknowledgments. Authors want to thank Francesco Galizzi for his contribution to the design of the platform.

\section{References}

1. Mamun, M.A.A., Yuce, M.R.: Sensors and systems for wearable environmental monitoring toward IoT-enabled applications: a review. IEEE Sensors J. 19(18), 7771-7788 (2019). https:// doi.org/10.1109/jsen.2019.2919352

2. Polhemus, A.M., et al.: Human-centered design strategies for device selection in mHealth programs: development of a novel framework and case study. JMIR Mhealth Uhealth 8(5), e16043 (2020). https://doi.org/10.2196/16043. PMID: 32379055, PMCID: 7243134

3. Locatelli, P., Alimonti, D.: Differentiating essential tremor and Parkinson's disease using a wearable sensor-a pilot study, In: 2017 7th IEEE International Workshop on Advances in Sensors and Interfaces (IWASI), Vieste, pp. 213-218 (2017). https://doi.org/10.1109/iwasi. 2017.7974254

4. Pedrana, A., Comotti, D., Locatelli, P., Traversi, G.: Development of a telemedicine-oriented gait analysis system based on inertial sensors. In: 2018 7th International Conference on Modern Circuits and Systems Technologies (MOCAST), Thessaloniki, pp. 1-4 (2018). https://doi.org/ $10.1109 /$ mocast.2018.8376592 